\title{
Growth Hormone Replacement Therapy in Patients without Adult Growth Hormone Deficiency: What Answers Do We Have So Far?
}

\author{
Karine Rucker ${ }^{1}$, Larissa Bianca Paiva Cunha de Sá1, Alberto Krayyem Arbex ${ }^{1,2}$ \\ ${ }^{1}$ Endocrinology Division, IPEMED Medical School, São Paulo, Brazil \\ ${ }^{2}$ Diabetology Department, Malteser Krankenhaus St. Franziskus-Hospital, Flensburg, Germany \\ Email:albertoarbex@gmail.com
}

How to cite this paper: Rucker, K., de Sá, L.B.P.C. and Arbex, A.K. (2017) Growth Hormone Replacement Therapy in Patients without Adult Growth Hormone Deficiency: What Answers Do We Have So Far? Health, 9, 799-810.

https://doi.org/10.4236/health.2017.95057

Received: February 27, 2017

Accepted: May 14, 2017

Published: May 18, 2017

Copyright $\odot 2017$ by authors and Scientific Research Publishing Inc. This work is licensed under the Creative Commons Attribution International License (CC BY 4.0).

http://creativecommons.org/licenses/by/4.0/

\begin{abstract}
Growth hormone (GH) and insulin-like growth factor 1 (IGF-1) have been suggested as "anti-aging" therapies, or for improving quality of life with aging. In this study, we focus on the actions of GH in the main organs and organ systems of the human body, like skeletal muscle, bones and brain, particularly in regard to data and research on the use of GH replacement therapy in adults without growth hormone deficiency, especially elderly patients. Several different studies have been carried out to show what the effects and side effects of GH replacement in healthy people and what would be the impact in quality of life and life span. In this review, we demonstrate what answers we have so far about the effects of GH replacement in many organs and systems in healthy people.
\end{abstract}

\section{Keywords}

Growth Hormone, Insulin-Like Growth Factor 1, Sarcopenia, Growth Hormone Replacement, Adult Growth Hormone Deficiency

\section{Introduction}

The global population is aging, and concurrently life expectancy is increasing. Aging is accompanied by organic changes, some of which are associated with the endocrine system. The function of the growth hormone (GH) and insulin-like growth factor 1 (IGF-1) axis declines with age, which may result in deleterious effects on body composition, the cardiovascular system, and cognition [1]. In the past decade, much evidence has indicated that the GH/IGF-1 system could play a role in many conditions and pathologies that commonly occur with aging, such as atherosclerosis, cardiovascular disease, cognitive decline, dementia, sar- 
copenia, and frailty [2].

The increase in average life expectancy has led to efforts to improve the quality of life in elderly people. After the peak of GH levels during puberty, the hormone levels in the GH/IGF-1 axis gradually decline, and by 60 years of age, many healthy individuals have 24-hour GH levels indistinguishable from those with adult growth hormone deficiency (AGHD) due to hypothalamic-pituitary lesions [3].

Secretion of GH and IGF-1 increases during puberty, and decreases accordingly with aging. Decreased GH and IGF-1 may be involved with aging-associated changes in body composition, structural function, and metabolism [4].

The similarities of physical and mental signs between decreased hormone levels in the GH/IGF-1 axis and due to AGHD, whose prevalence is $1 / 100,000$, according to Growth Hormone Research Society (GHRS), are evident, such as those that occur during normal aging, and the levels of sleep-induced GH are also decreased. Unlike patients with AGHD, both sleep and physical exercise persist as physiological stimuli for GH secretion in healthy elderly people [3].

The concept that GH could be prescribed and administered in patients without hypothalamic-pituitary disease is not recommended by endocrinology societies throughout the world, and is therefore still the topic of extensive discussion.

Research and studies have been carried out, and more work will continue as this is still a controversial subject that lacks consensus. Some advocate its therapeutic use in healthy elderly people, while others strongly oppose it. It remains unknown for the relationship between GH and sarcopenia, bone mineral density, the cardiovascular system, and cognition.

\section{GH/IGF-1 AXIS}

Growth hormone is a polypeptide produced primarily by the anterior pituitary. GH plays a major role in bone and soft tissue growth, particularly in the postnatal period [5].

GH regulates important physiological processes, including somatic growth and development, carbohydrate and lipid metabolism through direct activation of specific GH receptors, or indirectly through IGF-1, which is produced primarily in the liver in response to GH stimulus [6].

The regulation of $\mathrm{GH}$ production is controlled by two hypothalamic peptides: growth hormone-releasing hormone (GHRH), which stimulates hormone secretion by the anterior pituitary, and somatostatin (somatotropin release-inhibiting factor-SRIF), which inhibits this secretion [7].

GH/IGF-1 axis activity in humans is determined by several factors, including age and sex, but the mechanisms underlying these associations are uncertain. Both spontaneous and stimulated GH secretion in middle-aged adults is determined more by abdominal fat than by aging, so weight loss would help restore impaired GH secretion in obesity [8].

Growth hormone stimulates lipolysis, the breakdown of triglycerides, and their oxidation in cells. It is also involved in the regulation of blood glucose, de- 
creasing the ability of insulin to increase the uptake of glucose in peripheral tissues [9]. This hormone plays an important role in the regulation of anabolism and in the preservation of lean mass, and some of these anabolic effects are mediated by stimulation of lipolysis. Studies in humans have shown that GH reduces protein breakdown by about $30 \%$ during fasting, and this effect depends on the mobilization of free fatty acids from lipid stores [10].

GH/IGF-1 stimulates osteoblast proliferation and its differentiation in bones, inhibiting the activity of osteoclasts. In kidneys, it modulates 1-alpha-hydroxylase, which activates 25-hydroxycholecalciferol $(25(\mathrm{OH})$ it $\mathrm{D})$ and phosphate reabsorption. The effects of GH on muscle cell proliferation, muscle fiber size, and muscle fiber type depend on the action of IGF-1, while the effects on insulin sensitivity in muscle are independent of IGF-1 [11].

\section{GH/IGF-1 and Sarcopenia}

No other tissue undergoes more dramatic changes with aging than skeletal muscle [12]. Total body muscle mass is determined by the balance between synthesis and breakdown of proteins. Most anabolic muscle stimuli are through IGF-1, but also branched-chain amino acids (BCAA, such as leucine), exercise, and testosterone [13]. There is no clear association between IGF-1 levels and body composition, measurement of muscle strength, and physical performance in elderly men, because many epidemiological studies had negative findings [14]. For example, Kostka et al. [14] were able to detect an association between muscle strength and IGF-1 levels in elderly women, but not in elderly men.

IGF-1 stimulates the delivery of amino acids to muscles, suppresses proteases, and protects against damage after vigorous muscle contractions by attenuating inflammation and fibrosis, thereby facilitating muscle recovery after damage or injury [15].

Sarcopenia is a variable used to define frailty syndrome, which is highly prevalent in elderly people. Sarcopenia increases the risk of falls, fractures, disability, dependence, recurrent hospitalization, and mortality [16]. Frailty has recently been recognized as a distinct clinical entity, and it is described as a stage of decreased physiological reserves associated with increased risk of disability [14].

Sarcopenia is present in about $5 \%$ to $10 \%$ of people over 65 years of age. There are multiple causes, including decreased caloric intake, poor blood flow to the muscles, mitochondrial dysfunction, increased levels of proinflammatory cytokines, and low levels of anabolic hormones [16].

With aging, men have greater muscle loss due to low levels of GH/IGF-1 and testosterone. However, although the muscular loss is greater in men, it is important to emphasize the importance of sarcopenia in elderly women, since they have a longer life expectancy and high prevalence of functional limitations [17]. Aging is associated with a significant alteration in GH secretion patterns, and it has been shown that GH secretion decreases by $14 \%$ with each decade of age, and in a 70 -year-old man the average GH secretion has decreased more than $70 \%[18]$.

The importance of maintaining muscle mass and its physical and metabolic 
functions in elderly patients is well recognized [19].

Brioche, et al. demonstrated in a study published in 2014 with animal models (rodents) that GH supplementation to restore it to appropriate levels is a good intervention to preserve muscle mass in elderly individuals, and low doses of this hormone could be very useful to prevent aging-associated sarcopenia [20].

Recently, an association between hepatic steatosis and sarcopenia has been demonstrated. Several studies have shown that GH secretion is compromised in obese people, and GH and IGF-1 have been found to be involved in fatty infiltration of the liver [21]. On the other hand, the ectopic deposition of fat seems to be associated with reduced GH levels, as well as the decreased ability to produce GH with aging, which is one of the causes of body changes leading to excess adiposity and sarcopenia [22].

Probably the great interest in GH supplementation in elderly people is for treatment or prevention of sarcopenia and physical weakness. This concept was substantiated by the observation that two-years of GH replacement therapy in patients with AGHD restored muscle strength, although young patients had a superior outcome. Different dosages and duration of therapy, a lack of consistent evidence of AGHD, relatively small sample sizes, and different physical function tests make most of the studies difficult to compare, yet all suggest that GH supplementation would modestly increase lean mass [15].

There is an interest in the potential of GH replacement therapy associated with exercise to increase muscle strength and function in elderly men. Taaffe et al. demonstrated in a study, with 18 men, aged 65 - 82 years, with low levels of IGF-1, who completed resistance training for 14 weeks and used recombinant GH for 10 weeks, no increased muscle fibers (type I or II) or strength compared with placebo, although lean mass increased in those who underwent GH replacement therapy [14].

Several other studies corroborate these findings. That is, body composition is improved, and there is no difference in muscle strength or oxygen consumption during exercise in elderly patients undergoing recombinant GH replacement therapy as compared to controls. Some studies have shown discretely increased muscle strength and oxygen consumption in those who received testosterone in association with GH replacement therapy.

To date, GH is not formally indicated for either the prevention or treatment of adverse changes in body composition and for declined physical performance observed in elderly men. According to a consensus by the Endocrine Society (USA) and the Brazilian Society of Endocrinology and Metabolism, no consistent increase in muscle function or other measures of muscle performance have been demonstrated with GH therapy in elderly patients. Furthermore, no clear causal relationship between declining GH levels and the signs and symptoms attributed to these low levels has yet been established [14].

The practice of resistance exercise training is still the most effective intervention to increase muscle mass and strength in elderly people, and the greater acquisition of peak muscle mass is fundamental to delay the loss due to aging, and 
to cause a minor impact in the quality of life of elderly people [17].

\section{GH/IGF-1 and Bone Mineral Density}

Bone growth, development, and remodeling are modulated by several circulating hormones. The action of each of them differs throughout life [23].

The actions of GH/IGF-1 in longitudinal growth are already well described. However, this axis also regulates skeletal development and improves bone mineral density. Studies with rodents with axillary nerve dysfunction show a clear deterioration in bone health parameters depending on the expression of GH-induced IGF-1, which increases bone mineral density [24].

GH/IGF-1 in bone stimulates osteoblast differentiation and proliferation, inhibits osteoclast activity, and modulates the activity of 1-alpha-hydroxylase, which activates $25(\mathrm{OH}) \mathrm{D}$, and phosphate reabsorption [11].

Higher bone mineral density and a lower rate of osteoporotic fractures were consistently associated with high levels of IGF-1 in several cross-sectional studies carried out in men and women. In a small number of studies, this association was demonstrated in elderly men, while other studies have not found a positive association between bone mineral density and IGF-1 levels in elderly men [14].

Even in healthy patients, aging-associated decreases in serum GH and IGF-1 levels are related to a loss of muscle mass, bone loss, and increased risk of osteoporotic fractures. Bone responsiveness to IGF-1 also decreases with aging [25].

A great similarity is found between patients with AGHD and the phenotype of healthy patients with hormone depletion due to aging [14]. This also leads us to think how this parallel could be traced to osteoporosis and the loss of bone mineral density due to aging.

In patients with AGHD, where recombinant GH replacement therapy is indicated, several studies have shown that hormone replacement therapy for more than one year increases bone mineral density, more evidently in young patients. It was also observed in some randomized studies that the effect of recombinant GH is biphasic. That is, in the first six months of treatment, GH increased bone turnover, causing an initial loss of bone mass. Only after this initial period bone turnover decreases, and bone mineral density increases [26].

Van der Lely et al. carried out a study with patients over 60 years of age with accidental hip fracture $(n=111)$. The patients were randomized to receive $\mathrm{GH}$ for six weeks versus placebo, 24 hours after fracture, for 18 weeks. This study demonstrated that the benefit of GH replacement therapy was perceived in patients over 75 years of age, where patients resumed their pre-fracture activities, showing significantly better outcome than the placebo group in the same age group. The treatment was well tolerated and caused no significant harmful adverse effects [27].

A double-blind, randomized study conducted in Sweden by Krant et al. [28] followed menopausal women with osteoporosis $(n=80)$ for 10 years, all undergoing estrogenic hormone replacement therapy, where one group received re- 
combinant GH for 36 months and the other received placebo for 18 months. Both groups received calcium and vitamin D. Compared with a sample of the female population of the same age 50 - 70 years), it was demonstrated that the group that received GH had an improvement in bone mineral density, and the number of fractures decreased from $58 \%$ to $28 \%$ in this group, while in the control group fractures increased from $8 \%$ to $32 \%$. Quality of life, which was also measured in this study, showed no change during GH treatment or in the 10-year follow-up in both groups. The use of GH had no effect on the patients' glycemic profile or any significant side effects.

\section{H/IGF-1 and Cardiovascular System}

The amplitude of GH pulses decreases with aging, and it has been shown that GH-induced IGF-1 secretion also decreases, suggesting that a secondary factor is related to this decreased plasma IGF-1, due to resistance to GH action, which could also be associated with aging [29].

In the cardiovascular system, both excess GH (acromegaly) and AGHD increase cardiovascular morbidity and mortality. GH has an important effect on the maintenance of the structure and function of the heart in adults [30]. The GH/IGF-1 system interacts with the cardiovascular system and plays a role in regulating vascular tone and, consequently, peripheral resistance. Central effects of this hormone include its sympathetic actions, which also contribute to peripheral resistance [31].

A recent study in healthy people showed that GH directly increased blood flow in the forearm, in parallel to decreased peripheral vascular resistance. Furthermore, these changes have been suggested to be mediated by the stimulation of endothelial function through nitric oxide (NO) [32].

Many lines of evidence suggest that IGF-1 plays an important role in preventing the development of atherosclerosis and cardiovascular disease. A study that administered GH in patients with AGHD clearly demonstrated a significant reduction in risk factors for atherosclerosis, and early reversal of atherosclerotic alterations [33].

Several studies have described the cardiac structure in patients with AGHD and showed that patients with AGHD who received no hormone replacement therapy had decreased left ventricular mass and lower cardiac output, as well as reduced exercise capacity [34].

Several cardiovascular risk factors are associated with AGHD, including insulin resistance and unfavorable changes in lipid levels, including increased low density cholesterol. GH replacement therapy has normalized most cardiovascular risk factors in patients with hypopituitarism, although the effects of this replacement therapy on insulin sensitivity still remain controversial [32].

Vasan et al. showed in a prospective study that the ratio of IGF-1 levels was inversely related to the risk of congestive heart failure in elderly people without a history of a previous myocardial infarction. Several studies have demonstrated this same finding, correlating the severity of heart failure with low levels of 
IGF-1 [35].

Some studies have shown beneficial effects in patients with heart failure, both ischemic and idiopathic dilated cardiomyopathy, with improved hemodynamics when GH was added as maintenance therapy and short-term infusion [36] [37]. Other studies have not found this same result, which suggests that patients with heart failure are heterogeneous, and that IGF-1 is used differently in each study. Therefore, we have not found an adequate response regarding the use of GH in cardiac patients or as a treatment for improvement of cardiovascular risk factors.

\section{GH/IGF-1 and Cognition}

IGF-1 plays a critical role in the development and differentiation of the central nervous system. The growth and development of neurons in the dentate gyrus of the hippocampus are modulated by IGF-1. The dentate gyrus of the hippocampus is related to operational memory, regardless of the type of information involved in the task working memory/internal clock models and spatial learning cognitive map theory. As IGF-1 levels decrease with age, and cognition decreases as well, IGF-1 levels and dementia could be linked [38].

Several cross-sectional studies have linked reduced IGF-1 levels with cognitive decline, and favorable cognitive effects of growth hormone-releasing hormone (GHRH) have been reported in a controlled study in healthy elderly people [39].

Several epidemiological studies reported that IGF-1 levels are positively related to cognition, which comprises the ability to understand, verbal memory, working memory, to perform functions, and overall cognition in elderly individuals [40]. However, a recent study found no relationship between IGF-1 levels and cognition in middle-aged individuals [41]. These different results may be associated with differences in the populations studied (in some characteristics), measurement of IGF-1 levels, follow-up time, and dosages used.

Hence, in a cross-sectional study with 636 elderly men over 74 years of age, IGF-1 levels were independently and positively related to Mini-Mental Status Examination (MMSE) and verbal fluency, and insulin-like growth factor-binding protein 1 (IGFBP-1) level was inversely related to the MMSE [42]. This was confirmed in a meta-analysis involving 13 studies including 1982 subjects, which reported a relationship between IGF-1 levels and cognitive function in healthy elderly men [43].

In transgenic mice with Alzheimer's disease, increased IGF-1 levels decreased the accumulation of beta-amyloid, a characteristic disturbance in Alzheimer's disease [44]. In humans, supplementation with growth hormone-release hormone analogs improved cognition in healthy elderly people and those with mild cognitive impairment [39] [44].

\section{Possible Adverse Effects of GH Use in Patients without AGHD}

Studies in elderly people have shown an increased risk of side effects due to GH supplementation, such as carpal tunnel syndrome, gynecomastia, fluid retention, 
and, consequently, edema. There is also a concern of inducing insulin resistance with dose-dependent $\mathrm{GH}$ [45].

The circulating concentrations of IGF-1, IGF-2, and IGFBPs vary among healthy individuals, influenced by genetic factors and lifestyle, especially food habits. Population studies have shown a high risk of cancer in individuals with IGF-1 levels at the upper limit of normal range, although the risk of developing neoplasia does not seem to have increased in patients with AGHD undergoing replacement therapy [46].

The safety of GH replacement therapy in elderly individuals, such as the risk of developing cancer, remains unknown. Short-term studies with GH have not shown increased risk of malignancy, although long-term studies have not been available. In humans positive associations between circulating total IGF-I levels and cancer mortality have been found in many studies [47], while low total IGF-I levels have been associated with an increased risk for cardiovascular diseases and diabetes [48]. On the other hand, Rozing et al. showed that offspring of familial nonagenarians displayed similar IGF-I and IGFBP-3 levels compared to their partners [49].

In elderly people, not only the anabolic effects have greatly diminished but also the side effects have increased [50]. Many researchers found an increased incidence of side effects when GH was administered in elderly patients. In a group of elderly men that underwent GH replacement therapy for six months, Cohn, Rudman et al. reported a side-effect withdrawal rate of $43 \%$ compared to $9 \%$ in the placebo group. The most common symptoms were carpal tunnel syndrome, gynecomastia, and hyperglycemia [51]. Yarasheski et al. reported a similarly high incidence of carpal tunnel syndrome, edema, and arthralgia [52]. The diabetogenic effects of $\mathrm{GH}$ also seem to be increased in elderly people.

\section{Conclusions}

The effects of GH supplementation to treat aging-associated diseases have been studied, as well as the effect of GH/IGF-1 in several organs and tissues in adults. How physiological and natural is this decrease of GH/IGF-1? Further, how are the effects of aging to be treated? If decreased circulating levels of GH/IGF-1 are physiological, to what extent could this be corrected and treated to avoid aging-associated comorbidities?

We have defined indications for the use of GH in patients with AGHD, in both adults and children, and we know that GH is used off-label, such as, to improve lean mass and decrease fat mass. Although, to date, it has not been found that increased lean mass improves physical performance and consequently, muscle strength.

We are aware of several effects of the GH/IGF-1 axis in the cardiovascular system, as well as in lipid, carbohydrate, and glucose metabolism. Studies have shown beneficial effects on the cognition of elderly individuals, increased bone mineral density, including fracture recovery and prevention of new fractures, but these studies are still limited. 
Therefore, although some studies have shown positive results on the effect of GH replacement therapy in healthy elderly patients with AGHD due to aging, other studies have not demonstrated this effect or any meaningful effects to improve the quality of life, leaving many questions regarding supplementation unanswered. As the majority of studies, to date, have limited results for several reasons, such as dosage used, treatment time, and IGF-1 and GH dosing methods, we expect that more comprehensive studies, especially in the long term, will show whether the use of GH is safe or not. If safe, this further work will indicate what the benefits of GH therapy are. There is still a long way to go.

\section{References}

[1] Huayllas, M.K.P., Carvalhaes-Neto, N., Ramos, L.R. and Kater, C.E. (2001) Níveis séricos de hormônio de crescimento, fator de crescimento símile à insulina e sulfato de deidroepiandrosterona em idosos residentes na comunidade. Correlação com parâmetros clínicos. Arquivos Brasileiros de Endocrinologia \& Metabologia, 45, 157-166.

[2] Ceda, G.P., et al. (2005) Clinical Implications of the Reduced Activity of the GH-IGF1 Axis in Older Men. Journal of Endocrinological Investigation, 28, 96-100.

[3] Bronstein, M.D. (2003) Reposição de GH na "somatopausa": Solução ou problema? Arquivos Brasileiros de Endocrinologia \& Metabologia, 47, 323-330.

[4] Tanimoto, K., Hizuka, N., et al. (2008) The Influence of Age on the GH-IGF1 Axis in Patients with Acromegaly. European Journal of Endocrinology, 159, 375-379. https://doi.org/10.1530/EJE-08-0243

[5] Boguszewski, C.L. (2001) Genética molecular do Eixo GH-IGF1. Arquivos Brasileiros de Endocrinologia \& Metabologia, 45, 5-14.

[6] Perrini, S., Laviola, L., et al. (2010) The GH/IGF-1 Axis and Signaling Pathways in the Muscle and Bone: Mechanisms Underlying Age Related Skeletal Muscle Wasting and Osteoporosis. Journal of Endocrinology, 205, 201-210.

https://doi.org/10.1677/JOE-09-0431

[7] Naves, L.A. (2016) Acromegalia: Visão Geral in Vilar L. Endocrinologia Clinica, Guanabara Koogan, 6th Edition, 56-71.

[8] Vestergaard, P.F., et al. (2014) GH Signaling in Skeletal Muscle and Adipose Tissue in Healthy Human Subjects: Impact of Gender and Age. European Journal of Endocrinology, 171, 623-631. https://doi.org/10.1530/EJE-14-0538

[9] Ikeda, H. and Kudo, M. (2016) Long Term Follow Up Results of Growth Hormone Therapy for Patients with Adults Growth Hormone Deficiency. Hormones, 15, 4553.

[10] Vendelbo, M.H., Christensen, B. and Gronbaek, S.B. (2015) GH Signaling in Human Adipose and Muscle Tissue during "Feast and Famine": Amplification of Exercise Stimulation Following Fasting Compared to Glucose Administration. European Journal of Endocrinology, 173, 283-290. https://doi.org/10.1530/EJE-14-1157

[11] Girgls, C.M., Mokbel, N. and DiGirolamo, D.J. (2014) Therapies for Musculoskeletal Disease: Can We Treat Two Birds with One Stone? Current Osteoporosis Reports, 12, 142-153. https://doi.org/10.1007/s11914-014-0204-5

[12] Rosenberg, I.H. (1997) Sarcopenia: Origins and Clinical Relevance. Journal of Nutrition, 127, 990S-991S.

[13] All, S. and Gracia, J. (2014) Sarcopenia, Cachexia and Aging: Diagnosis, Mechan- 
isms and Therapeutic Options-A Mini-Review. Gerontology, 60, 294-305.

[14] Giannoulis, M.G., Martin, F.C., et al. (2012) Hormone Replacement Therapy and Physical Function in Healthy Older Men. Time to Talk about Hormones? Endocrine Reviews, 33, 314-377. https://doi.org/10.1210/er.2012-1002

[15] Sattler, F.R. (2013) Growth Hormone in the Aging Male. Best Practice \& Research. Clinical Endocrinology \& Metabolism, 27, 541-555. https://doi.org/10.1016/j.beem.2013.05.003

[16] Morley, J.E., Anker, S.D. and Von Hachling, S. (2014) Prevalence, Incidence and Clinical Impact of Sarcopenia: Facts, Numbers and Epidemiology-Update 2014. Journal of Cachexia, Sarcopenia and Muscle, 5, 253-259. https://doi.org/10.1007/s13539-014-0161-y

[17] Silva, T.A.A., et al. (2006) Sarcopenia associada ao envelhecimento: Aspectos Etiológicos e Opções Terapêuticas. Revista Brasileira de Reumatologia, 46, 391-397.

[18] Veldhuis, J.D., Liem, A.Y., South, S., Weltman, A., Weltman, J., Clemmons, D.A., Abbott, R., Mulligan, T., Johnson, M.L. and Pincus, S. (1995) Differential Impact of Age, Sex Steroid Hormones, and Obesity on Basal versus Pulsatile Growth Hormone Secretion in Men as Assessed in an Ultrasensitive Chemiluminescence Assay. The Journal of Clinical Endocrinology and Metabolism, 80, 3209-3222.

[19] Wolfe, R.R. (2006) The Underappreciated Role of Muscle in Health and Disease. The American Journal of Clinical Nutrition, 84, 475-482.

[20] Brioche, T., et al. (2014) Growth Hormone Replacement Therapy Prevents Sarcopenia by a Dual Mechanism: Improvement of Protein and of Antioxidant Defenses. The Journals of Gerontology Series A Biological Sciences and Medical Sciences, 69, 1186-1198.

[21] Takahashi, Y. (2012) Essential Roles of Growth Hormone (GH) and Insulin-Like Growth Factor in the Liver. Endocrine Journal, 59, 955-962. https://doi.org/10.1507/endocrj.EJ12-0322

[22] Poggiogalle, E., et al. (2016) Fatty Liver Index Associates with Relative Sarcopenia an GH/IGF-1 Status in Obese Subjects. PLoS ONE, 11, e0145811. https://doi.org/10.1371/journal.pone.0145811

[23] Southmay, E.A. and de Souza, M.J. (2016) A Summary of Influence of Exogenous Estrogen Administration across the Lifespan on the GH/IGF-1 Axis and Implications for Bone Health. Growth Hormone and IGF Research, 32, 2-13.

[24] Devesa, J., Almenglo, C. and Devesa, P. (2016) Multiple Effects of Growth Hormone in the Body: Is It Really the Hormone for Growth? Clinical Medicine Insights. Endocrinology and Diabetes, 9, 47-71. https://doi.org/10.4137/CMED.S38201

[25] Zhao, H.Y., Liu, J.M., Ning, G., Zhao, Y.J., Chen, Y., Sun, L.H., et al. (2008) Relationships between Insulin-Like Growth Factor-I (IGF-I) and OPG, RANKL, Bone Mineral Density in Healthy Chinese Women. Osteoporosis International, 19, 221226. https://doi.org/10.1007/s00198-007-0440-y

[26] Maya, B., Klibanski, A. and Tritos, N.A. (2014) Effects of Recombinant Human Growth Hormone Therapy on Bone Mineral Density in Adults with Growth Hormone Deficiency: A Meta-Analysis. The Journal of Clinical Endocrinology and Metabolism, 99, 852-860. https://doi.org/10.1210/jc.2013-3921

[27] Van der Lely, A.J., et al. (2000) Use of Human GH in Elderly Patients with Accidental Hip Fracture. European Journal of Endocrinology, 143, 585-592. https://doi.org/10.1530/eje.0.1430585

[28] Krantz, E., Trimpou, P. and Landin-Wilhelmsen, K. (2010) Effect of Growth Hormone Treatment of Fractures and Quality of Life in Postmenopausal Osteoporosis: 
A 10-Year Follow-Up Study. The Journal of Clinical Endocrinology and Metabolism, 100, 3251-3259. https://doi.org/10.1210/jc.2015-1757

[29] Khan, A.S., et al. (2002) Growth Hormone, Insulin-Like Growth Factor-1 and the Aging Cardiovascular System. Cardiovascular Research, 54, 25-35. https://doi.org/10.1016/S0008-6363(01)00533-8

[30] Sacca, L., Cittadini, A. and Fazio, S. (1994) Growth Hormone and the Heart. Endocrine Reviews, 15, 555-573. https://doi.org/10.1210/edrv-15-5-555

[31] Sverrisdotir, Y., et al. (1998) Intense Sympathetic Nerve Activity in Adults with Hypopituitarism and Untreated Growth Hormone Deficiency. The Journal of Clinical Endocrinology and Metabolism, 83, 1881-1885.

[32] Isgaard, J., Arcopinto, M., Karason, K. and Cittadini, A. (2015) GH and the Cardiovascular System: An Update on Topic at Heart. Endocrine, 48, 25-35. https://doi.org/10.1007/s12020-014-0327-6

[33] Gola, M., Bonadonna, S., Doga, M. and Giustina, A. (2005) Clinical Review: Growth Hormone and Cardiovascular Risk Factors. The Journal of Clinical Endocrinology and Metabolism, 90, 1864-1870. https://doi.org/10.1210/jc.2004-0545

[34] Cittadini, A., et al. (1994) Impaired Cardiac Performance in GH Deficient Adults and Its Improvement after GH Replacement. American Journal of Physiology, 267, 219-225.

[35] Vasan, R.S., Sullivan, L.M., D’Agostino, R.B., Roubenoff, R., Harris, T., Sawyer, D.B., Levy, D. and Wilson, P.W. (2003) Serum Insulin-Like Growth Factor I and Risk for Heart Failure in Elderly Individuals without a Previous Myocardial Infarction: The Framingham Heart Study. Annals of Internal Medicine, 139, 642-648. https://doi.org/10.7326/0003-4819-139-8-200310210-00007

[36] Genth-Zotz, S., Zotz, R., Geil, S., Voigtlander, T., Meyer, J. and Darius, H. (1999) Recombinant Growth Hormone Therapy in Patients with Ischemic Cardiomyopathy. Effects on Hemodynamics, Left Ventricular Function and Cardiopulmonary Exercise Capacity. Circulation, 99, 18-21. https://doi.org/10.1161/01.CIR.99.1.18

[37] Volterrani, M., Desenzani, P., Lorusso, R., d'Aloia, A., Manelli, F. and Giustina, A. (1997) Haemodynamic Effects of Intravenous Growth Hormone in Congestive Heart Failure. The Lancet, 349, 1067-1068. https://doi.org/10.1016/S0140-6736(97)24015-8

[38] Deijen, J.B., Arwert, L.I. and Drent, M.L. (2011) The GH/IGF-I Axis and Cognitive Changes across a 4-Year Period in Healthy Adults. ISRN Endocrinology, 2011, Article ID: 249421.

[39] Vitiello, M.V., Moe, K.E., Merriam, G.R., Mazzoni, G., Buchner, D.H. and Schwartz, R.S. (2006) Growth Hormone Releasing Hormone Improves the Cognition of Healthy Older Adults. Neurobiology of Aging, 27, 318-323. https://doi.org/10.1016/j.neurobiolaging.2005.01.010

[40] Tumati, S., et al. (2016) Association between Cognition and Serum Insulin-LikeGrowth-Factor-1 in Middle-Aged and Older Men: An 8-Year Follow-Up Study. PLOS ONE, 11, e0154450.

[41] Licht, C.M., van Turenhout, L.C., Deijen, J.B., Koppes, L.L., van Mechelen, W., Twisk, J.W., et al. (2014) The Association between IGF-1 Polymorphisms, IFG-1 Serum Levels and Cognitive Function in Healthy Adults: The Amsterdam Growth and Healthy Longitudinal Study. International Journal of Endocrinology, 2014, Article ID: 181327. https://doi.org/10.1155/2014/181327

[42] Delaimy, W.K., von Muhlen, D. and Barrett-Connor, E. (2009) Insulin-Like Growth Factor-1, Insulin-Like Growth Factor Binding Protein-1, and Cognitive Function in 
Older Men and Women. Journal of the American Geriatrics Society, 57, 1441-1446. https://doi.org/10.1111/j.1532-5415.2009.02343.x

[43] Arwert, L.I., Deijen, J.B. and Drent, M.L. (2005) The Relation between Insulin-Like Growth Factor I Levels and Cognition in Healthy Elderly: A Meta-Analysis. Growth Hormone \& IGF Research, 15, 416-422. https://doi.org/10.1016/j.ghir.2005.09.001

[44] Carro, E., Trejo, J.L., Gerber, A., et al. (2006) Therapeutic Actions of Insulin-Like Growth Factor I on APP/PS2 Mice with Severe Brain Amyloidosis. Neurobiology of Aging, 27, 1250-1257. https://doi.org/10.1016/j.neurobiolaging.2005.06.015

[45] Liu, H., Bravata, D.M., Olkin, I., et al. (2007) Systematic Review: The Safety and Efficacy of Growth Hormone in the Healthy Elderly. Annals of Internal Medicine, 146, 104-115. https://doi.org/10.7326/0003-4819-146-2-200701160-00005

[46] Spinola e Castro, A.M., de São Paulo, S.P. and Guerra-Júnior, G. (2005) GH/IGF-1 e neoplasia: O que há de novo nessa associação. Arquivos Brasileiros de Endocrinologia \& Metabologia, 49, 833-842.

[47] Renehan, A.G., Zwahlen, M., Minder, C., O’Dwyer, S.T., Shalet, S.M. and Egger, M. (2004) Insulin-Like Growth Factor (IGF)-I, IGF Binding Protein-3, and Cancer Risk: Systematic Review and Meta-Regression Analysis. The Lancet, 363, 1346-1353. https://doi.org/10.1016/S0140-6736(04)16044-3

[48] Janssen, J.A. and Lamberts, S.W. (2004) Igf-I and Longevity. Logo Hormone Research in Paediatrics, 62, 104-109. https://doi.org/10.1159/000080508

[49] Rozing, M.P., Westendorp, R.G., Frölich, M., de Craen, A.J., Beekman, M., Heijmans, B.T., Mooijaart, S.P., Blauw, G.J., Slagboom, P.E. and van Heemst, D., Leiden Longevity Study (LLS) Group (2009) Human Insulin/IGF-1 and Familial Longevity at Middle Age. Aging (Albany NY), 1, 714-722.

https://doi.org/10.18632/aging.100071

[50] Brost, S.E. (2004) Interventions for Sarcopenia and Muscle Weakness in Older People. Age and Ageing, 33, 548-555. http://ageing.oxfordjournals.org

[51] Cohn, L., Feller, A.G., Draper, M.W., Rudman, I.W. and Rudman, D. (1993) Carpal Tunnel Syndrome and Gynecomastia during Growth Hormone Treatment of Elderly Men with Low Circulating IGF-I Concentrations. Clinical Endocrinology, 39, 417-425. https://doi.org/10.1111/j.1365-2265.1993.tb02388.x

[52] Yarasheski, K.E. and Zachwieja, J.J. (1993) Growth Hormone Therapy for the Elderly: The Fountain of Youth Proves Toxic. JAMA, 270, 1694-1694.

https://doi.org/10.1001/jama.1993.03510140054027 
Submit or recommend next manuscript to SCIRP and we will provide best service for you:

Accepting pre-submission inquiries through Email, Facebook, LinkedIn, Twitter, etc. A wide selection of journals (inclusive of 9 subjects, more than 200 journals)

Providing 24-hour high-quality service

User-friendly online submission system

Fair and swift peer-review system

Efficient typesetting and proofreading procedure

Display of the result of downloads and visits, as well as the number of cited articles Maximum dissemination of your research work

Submit your manuscript at: http://papersubmission.scirp.org/

Or contact health@scirp.org 